\title{
CONSTRUCTING TILTING MODULES
}

\author{
OTTO KERNER AND JAN TRLIFAJ
}

\begin{abstract}
We investigate the structure of (infinite dimensional) tilting modules over hereditary artin algebras. For connected algebras of infinite representation type with Grothendieck group of rank $n$, we prove that for each $0 \leq i<n-1$, there is an infinite dimensional tilting module $T_{i}$ with exactly $i$ pairwise non-isomorphic indecomposable finite dimensional direct summands. We also show that any stone is a direct summand in a tilting module. In the final section, we give explicit constructions of infinite dimensional tilting modules over iterated one-point extensions.
\end{abstract}

The study of finite dimensional tilting modules of projective dimension at most one over finite dimensional algebras was initiated by Brenner and Butler [11] and continued by Happel and Ringel [17]. Since then, many variations of this concept have been introduced and used successfully, for example: Tilting modules of higher projective dimension, tilting modules over rings, tilting complexes in derived categories, tilting objects in hereditary categories or in cluster categories. In this paper, we use the term tilting module as follows: Let $R$ be a ring and $T$ be a right $R$-module. Then $T$ is a tilting module provided that (T1) p.dim $T \leq 1$, (T2) $\operatorname{Ext}_{R}^{1}\left(T, T^{(I)}\right)=0$ for any set $I$, and (T3) there is a short exact sequence $0 \rightarrow R \rightarrow T_{0} \rightarrow T_{1} \rightarrow 0$ where $T_{0}$ and $T_{1}$ are direct summands in a direct sum of (possibly infinitely many) copies of $T$. Equivalently, $T$ is tilting if and only if $\operatorname{Gen}(T)=\{T\}^{\perp},[13]$.

Here, $\operatorname{Gen}(T)$ denotes the class of all homomorphic images of direct sums of copies of $T$, and, for a class of modules $\mathcal{C}$,

$$
\mathcal{C}^{\perp}=\operatorname{KerExt}_{R}^{1}(\mathcal{C},-)=\left\{M \in \operatorname{Mod}-R \mid \operatorname{Ext}_{R}^{1}(C, M)=0 \text { for all } C \in \mathcal{C}\right\} .
$$

If $T$ is a tilting module, then $\{T\}^{\perp}$ is a torsion class in Mod- $R$, the tilting class generated by $T$. If $T^{\prime}$ is another tilting module, then $T$ is said to be equivalent to $T^{\prime}$ if $\{T\}^{\perp}=\left\{T^{\prime}\right\}^{\perp}$.

Though our definition of a tilting module allows infinitely generated modules, there is an implicit finiteness property connected with tilting, recently proved by Bazzoni and Herbera in [9, Theorem 2.4]. Namely, any tilting module $T$ is of finite type, that is, there exists a set $\mathcal{S}$ consisting of finitely presented modules of projective dimension at most 1 such that $\mathcal{S}^{\perp}=\{T\}^{\perp}$.

Received by the editors November 29, 2005

2000 Mathematics Subject Classification. Primary 16E30, 16G10, 16G30; Secondary 16D50, $18 \mathrm{E} 40$.

This research was done during visits of the first author to Charles University, Prague, and of the second author to Heinrich Heine University, Düsseldorf, within the bilateral university exchange program.

The second author was supported by grants GAUK 448/2004/B-MAT and MSM 0021620839. 
It has been shown in [25, Theorem 2.1] that for an artin algebra $A$ there is a bijection between the set of equivalence classes of tilting $A$-modules of finite type and the set of torsion classes $\{\mathcal{T} \mid \mathrm{D}(A) \in \mathcal{T}\}$ in mod- $A$ where $\mathrm{D}(A)$ denotes the injective cogenerator in mod- $A$. The bijection is defined by $T \mapsto\{T\}^{\perp} \cap \bmod -A$.

Combining these two results, we get for an artin algebra $A$ a bijection between equivalence classes of tilting modules in $\operatorname{Mod}-A$ and torsion classes in $\bmod -A$ containing all injective modules. We therefore call such torsion classes $\mathcal{T}$ in mod- $A$ tilting torsion classes. (This notion generalizes the classical case where only finite dimensional tilting modules were considered, and hence the extra condition of $\mathcal{T}$ being generated by a single module had to be assumed; cf. [3], [19].)

Our first goal here is to investigate direct summands of tilting modules, in particular, the finite dimensional ones, and to use these investigations to construct tilting modules with various additional properties.

We will show that indecomposable finite dimensional direct summands of a tilting module $T \in \operatorname{Mod}-A$ coincide with the indecomposable Ext-projective modules in $\mathcal{T}=\{T\}^{\perp} \cap \bmod -A$. Then we will study the open problem of the number of indecomposable Ext-projective modules in tilting torsion classes in $\bmod -A$ for hereditary artin algebras, and prove

Theorem A. Let $H$ be a connected hereditary artin algebra of infinite representation type with $n$ simple modules.

(1) If $T$ is a tilting $H$-module with at least $n-1$ pairwise non-isomorphic finite dimensional indecomposable direct summands, then $T$ is equivalent to a finite dimensional tilting $H$-module $\hat{T}$ (so that $T$ has exactly $n$ such direct summands).

(2) For any natural number $i$ with $0 \leq i \leq n-2$, there exists an infinite dimensional tilting $H$-module $T_{i}$ with exactly $i$ pairwise non-isomorphic finite dimensional indecomposable direct summands.

The proof of Theorem A will be presented in Section 2 .

As a direct consequence, we obtain

Corollary. Let $H$ be a connected hereditary artin algebra with $n$ simple modules and $\mathcal{T}$ a tilting torsion class in mod- $H$.

(a) If $H$ is representation finite, then $\mathcal{T}$ contains $n$ pairwise non-isomorphic indecomposable Ext-projective modules.

(b) If $H$ is representation infinite and $0 \leq i \leq n$ with $i \neq n-1$, then there exists a tilting torsion class in mod-H with $i$ pairwise non-isomorphic indecomposable Ext-projective modules.

It should be mentioned that the Ext-projective torsion modules are central in the construction of torsion pairs in [4].

In Section 3, we study the problem of which modules in Mod- $A$ occur as direct summands of tilting $A$-modules. Of course, any such module has projective dimension $\leq 1$, and no self-extensions.

Until now, only partial cases were considered. In [13], a module $X$ is called partial tilting if $\operatorname{Gen}(X)=\{X\}^{\perp}$ and $\{X\}^{\perp}$ is a torsion class in Mod- $A$, that is, if $X$ can be completed to a tilting module $T$ by the Bongartz construction. 
Restricting to right hereditary right artinian rings, we show

Theorem B. Let $H$ be a right hereditary right artinian ring and $X$ be an $H$ module with $\operatorname{Ext}_{H}^{1}\left(X, X^{(I)}\right)=0$ for every set $I$. If $X$ is finitely generated over its endomorphism ring, then $X$ is a direct summand in a tilting $H$-module.

The assumption that $X$ is finitely generated over its endomorphism ring, frequently called finendo, is quite natural: by [13, Proposition 2.5.], each tilting module is finendo.

Let $H$ be a connected hereditary algebra. The endofinite modules, that is, the modules of finite length over their endomorphism rings, clearly are finendo. Since any endofinite module $X$ is $\Sigma$-pure-injective (see $[14,26]$ ) the pure embedding $X^{(I)} \rightarrow X^{I}$ splits, hence $\operatorname{Ext}_{H}^{1}(X, X)=0$ implies in this case the stronger condition of $\operatorname{Ext}_{H}^{1}\left(X, X^{(I)}\right)=0$. Since endofinite modules are direct sums of indecomposable endofinite modules $[14,4.5]$ with local endomorphism rings and nilpotent radical $[14,4.2,4.4]$, we may restrict to indecomposable endofinite modules $X$ with $\operatorname{Ext}_{H}^{1}(X, X)=0$. Following Schofield, such an $H$-module $X$ is called a stone. Stones are studied in [15] in the case where the ground-field $k$ is algebraically closed: There always exist infinite dimensional stones when $H$ is connected and representation-infinite. They are in 1-1 correspondence with imaginary indivisible Schur roots, whereas finite dimensional stones correspond to real Schur roots. The correspondence is defined as follows: Let $X$ be a stone and $E=\operatorname{End}_{H}(X)$. Then $E$ is a division algebra; see [15]. If $\left(P_{1}, \ldots, P_{n}\right)$ is a compete family of pairwise non-isomorphic indecomposable projective $H$-modules, then the assignment $X \mapsto\left(\operatorname{dim}_{E} \operatorname{Hom}_{H}\left(P_{i}, X\right)\right)_{1 \leq i \leq n}$ defines this bijection. Since $\operatorname{dim}_{E} \operatorname{Hom}_{H}\left(P_{i}, X\right)$ is finite and each finite dimensional $H$-module $M$ has a finite dimensional projective cover, $\operatorname{dim}_{E} \operatorname{Hom}_{H}(M, X)$ is also finite, for $M$ finite dimensional.

If $H$ is tame, there is a unique imaginary indivisible Schur root, and the generic module, $G$, is the unique infinite dimensional stone (originally studied in [31]). If $H$ is wild, there always exist infinitely many such roots; consequently, there exist infinitely many pairwise non-isomorphic infinite dimensional stones. We immediately get from Theorem B:

Corollary. Let $H$ be a hereditary algebra, and $X$ be a stone. Then $X$ is a direct summand in a tilting $H$-module.

The proof of Theorem B is done in several steps. One of these steps (Proposition 3.3 ) deals with the problem of when a tilting $R / I$-module $\bar{T}$, where $I$ is an idempotent ideal in $R$, can be completed to a tilting $R$-module $T$ with $\{T\}^{\perp}=\{\bar{T}\}^{\perp}$. This situation is studied again in the special case when $B$ is a finite dimensional $k$-algebra, $\bar{T} \in$ Mod- $B$ a tilting $B$-module and $A$ an iterated one-point extension of $B$. We obtain rather explicit results concerning this case in Section 4.

For background on representation theory of finite dimensional modules, we refer to $[5,6,32]$, of infinite dimensional modules to $[15,26,31]$, and on wild hereditary algebras to [23].

\section{Preliminaries}

For a commutative artinian ring $k$, a $k$-algebra $A$ is called an artin algebra if it is finitely generated as a $k$-module. Additionally, we will assume that $A$ is a faithful $k$-module, and that $A$ is connected. This means that 0 and 1 are the only central idempotents in $A$, in particular, $k$ is a local ring. 
By Mod- $A$, we denote the category of all (right $A$-) modules, and by $\bmod -A$ the subcategory of all finitely presented modules. ind- $A$ will denote a (fixed) representative set of the class of all finitely generated indecomposable modules. If $I$ is the injective hull of the simple $k$-module, then the functor $\mathrm{D}=\operatorname{Hom}_{k}(-, I)$ defines a duality between mod- $A$ and $A$-mod. Also, $\tau_{A}=\mathrm{DTr}$ and $\tau_{A}^{-}=\operatorname{TrD}$ denote the Auslander-Reiten translations in $\bmod -A$.

The Auslander-Reiten quiver, $\Gamma(A)$, is a directed graph whose set of vertices is ind- $A$, and whose arrows are induced by the Auslander-Reiten sequences $0 \rightarrow \tau X \rightarrow$ $E \rightarrow X \rightarrow 0$ for $X \in$ ind- $A$ non-projective, and by the embeddings $\operatorname{rad} X \subseteq X$ for $X \in$ ind- $A$ projective. For more details, see [6].

Moreover, if $A$ is hereditary, then $k$ is a field, hence $A$ is a finite dimensional $k$-algebra and $\tau_{A}^{-}=\operatorname{Ext}_{A}^{1}(\mathrm{D}(A),-)$ and $\tau_{A}=\operatorname{DExt}_{A}^{1}(-, A) \cong \operatorname{Tor}_{1}^{A}(\mathrm{D}(A),-)$ are endo-functors on mod- $A$.

Assume $A$ is hereditary and representation-infinite. Then $\Gamma(A)$ is partitioned into three types of modules: a module $X \in$ ind- $A$ is preprojective (preinjective) if $\tau^{m} X=0\left(\tau^{-m} X=0\right)$ for some $m \geq 0 ; X$ is regular if $\tau^{m} \tau^{-m} X \cong X$ for all integers $m$. A module $M \in \bmod -A$ is preprojective (preinjective, and regular) if either $M=$ 0 , or each indecomposable direct summand of $M$ is isomorphic to a preprojective (preinjective, and regular) module in ind- $A$. The set of all $M \in \bmod -A$ that are preprojective (preinjective, and regular) will be denoted by $\mathcal{P}(\mathcal{I}$, and $\mathcal{R})$. To avoid ambiguity, occasionally for example $\mathcal{P}(A)$ instead of just $\mathcal{P}$ will be written.

The Auslander-Reiten quiver $\Gamma(A)$ consists of infinitely many (connected) components: one preprojective component whose vertices are the elements of $\mathcal{P} \cap$ ind $-A$, one preinjective component with the elements of $\mathcal{I} \cap$ ind- $A$ as vertices, and an infinite set of regular components (with vertices in $\mathcal{R} \cap$ ind- $A$ ).

If $A$ is tame hereditary, all regular components are tubes, all of them homogeneous, up to finitely many. If $A$ is wild hereditary, all regular components are of type $Z A_{\infty}$. In both cases, the modules at the border of the regular components are called quasi-simple. If $Y$ is an arbitrary indecomposable module contained in a regular component $\mathcal{C}$, there exists a unique quasi-simple module $X$ in $\mathcal{C}$ and a chain of irreducible monomorphisms

$$
X=X(1) \rightarrow X(2) \rightarrow \cdots \rightarrow X(r)=Y,
$$

which we will consider as inclusions. The number $r$ is called the quasi-length of $Y$, and $X(i) / X(i-1) \cong \tau^{-i+1} X$ holds for $1<i \leq r$; see [30].

Dually, there exists a unique quasi-simple module $Z$ in $\mathcal{C}$ and a chain of irreducible epimorphisms of the same length $r$

$$
Y=(r) Z \rightarrow(r-1) Z \rightarrow \cdots \rightarrow(1) Z=Z .
$$

Given an abelian category $\mathcal{A}$, we call a pair $(\mathcal{T}, \mathcal{F})$ of classes of objects in $\mathcal{A}$ a torsion pair if $\operatorname{Hom}(\mathcal{T}, \mathcal{F})=0$, and both classes are maximal with respect to this property. This means that for any object $M \in \mathcal{A}$, there is a short exact sequence, called the canonical short exact sequence

$$
0 \rightarrow t(M) \rightarrow M \rightarrow f(M) \rightarrow 0,
$$

with $t(M) \in \mathcal{T}$ and $f(M) \in \mathcal{F}$.

An object $P \in \mathcal{T}$ is called Ext-projective (in $\mathcal{T})$, provided $\operatorname{Ext}_{\mathcal{A}}^{1}(P, \mathcal{T})=0$, that is, $\mathcal{T} \subseteq\{P\}^{\perp}$. 
If $H$ is a finite dimensional hereditary algebra with $n$ simple modules and $X$ is a finite dimensional $H$-module with $\operatorname{Ext}_{H}^{1}(X, X)=0$, we consider the full subcategory $\{X\}^{\perp \geq 0}$ of Mod- $H$, defined by the modules $\left\{M \mid \operatorname{Hom}_{H}(X, M)=\right.$ $\left.0=\operatorname{Ext}_{H}^{1}(X, M)\right\}$. It is easy to check that $\{X\}^{\perp_{\geq 0}}$ is an exact and extension closed abelian subcategory of Mod- $H$. It is well known that for $X$ indecomposable, $\{X\}^{\perp \geq 0}$ is equivalent to a module category Mod- $C$, where $C$ is a finite dimensional hereditary algebra with $n-1$ simple modules. Indeed, if $X=e H$ is indecomposable projective, then $\{X\}^{\perp_{\geq 0}} \cong \operatorname{Mod}-(H / H e H)$. If $X$ is not projective, and $0 \rightarrow H \rightarrow Y \rightarrow X^{r} \rightarrow 0$ is the Bongartz universal short exact sequence [10], then $Y$ is a finite dimensional projective generator in $\{X\}^{\perp \geq 0}$, hence $X^{\perp \geq 0}$ is equivalent to $\operatorname{Mod}_{-\operatorname{End}_{H}}(Y)$.

More generally, if $X^{\prime}$ is a finite dimensional partial tilting module with $r$ pairwise non-isomorphic indecomposable direct summands, then $\left\{X^{\prime}\right\}^{\perp \geq 0}$ is equivalent to Mod- $C^{\prime}$, where $C^{\prime}$ is finite dimensional hereditary with $n-r$ pairwise nonisomorphic simple modules. Indeed, let $0 \rightarrow H \rightarrow E \rightarrow X^{\prime r} \rightarrow 0$ be the Bongartz universal short exact sequence. Then the factor-module $Y$ of $E$ by the $X$-trace of $E$ is a small projective generator in $\left\{X^{\prime}\right\}^{\perp_{\geq 0}}$, hence $\left\{X^{\prime}\right\}^{\perp_{\geq 0}} \cong \operatorname{Mod}-C^{\prime}$, where $C^{\prime}=\operatorname{End}_{H}(Y)$; see [16, Proposition 3.8]. Especially, if $X^{\prime}$ has $n-1$ pairwise nonisomorphic indecomposable direct summands, then $C^{\prime}$ is a hereditary artin algebra with one simple module $S$, which means $C^{\prime}$ is a full matrix ring over the division algebra $K=\operatorname{End}(S)$. We refer to [16] for more details on perpendicular categories.

\section{Finite dimensional DiRECT SUmmands of tilting MOdUles}

The first aim of this section is to study finitely generated indecomposable direct summands of a tilting module $T$ in $\operatorname{Mod}-A$, for $A$ an artin algebra. For this purpose, we consider the torsion class $\mathcal{T}=\{T\}^{\perp} \cap \bmod -A$ in mod- $A$, and its corresponding torsion free class $\mathcal{F}$.

Lemma 2.1. Let $A$ be an artin algebra and $T$ a tilting $A$-module. A finitely generated $A$-module $X$ is in add $T$ if and only if $X$ is Ext-projective in $\mathcal{T}$.

Proof. If $X \in \operatorname{add} T$, then $X$ is Ext-projective in $\{T\}^{\perp}$, hence also in $\mathcal{T}$.

Conversely, let $X$ be Ext-projective in $\mathcal{T}$. Then $\tau_{A} X$ is in $\mathcal{F}$ by $[7,19]$. Since the injective cogenerator $D A$ is a torsion module, $\operatorname{Hom}_{A}\left(D A, \tau_{A} X\right)=0$ follows. Hence p.dim $X \leq 1$ by $[32,2.4]$. Combining [9, Theorem 2.4] and [25, Theorem 2.1], we get $\{T\}^{\perp}=\left(\tau_{A}^{-} \mathcal{F}\right)^{\perp}$. Since $X \in \tau_{A}^{-} \mathcal{F}$, we have $X \in\{T\}^{\perp} \subset\{X\}^{\perp}$. Consequently $X \in \operatorname{add} T$ by $[13$, Lemma 2.2$]$.

The following lemma is well known:

Lemma 2.2. Let $R$ be a ring and $X$ an $R$-module with a local endomorphism ring. If $X$ is a direct summand of $Y=\bigoplus_{i=1}^{r} Y_{i}$, then $X$ is a direct summand of some direct summand $Y_{i_{0}}$.

Proposition 2.3. Let $A$ be an artin algebra and $T$ a tilting $A$-module. If $\left\{X_{i} \mid 1 \leq\right.$ $i \leq r\}$ is a set of pairwise non-isomorphic indecomposable Ext-projective modules in $\mathcal{T}$, then $\bigoplus_{i} X_{i}$ is a direct summand of $T$.

Proof. By Lemma $2.1 X_{i}$ is in addT with a local endomorphism ring. Therefore, by Lemma 2.2, $X_{i}$ is a direct summand of $T$, for each $i$.

The proof now is done by induction. $X_{1}$ is a direct summand of $T$. Assume $T=\left(\bigoplus_{j=1}^{t-1} X_{j}\right) \oplus Y$ for $1<t \leq r$. Since $X_{t}$ is a direct summand of $T$, but not a 
direct summand of the indecomposable modules $X_{j}$, for $1 \leq j<t$, again by Lemma $2.2 X_{t}$ is a direct summand of $Y$. Consequently $\bigoplus_{j=1}^{t} X_{j}$ is a direct summand of $T$.

Proof of Theorem A(1). Let $X_{1}, \ldots, X_{n-1}$ be the pairwise non-isomorphic indecomposable finite dimensional direct summands of $T$ and $X=\bigoplus_{i} X_{i}$. Clearly, $\{T\}^{\perp} \subset\{X\}^{\perp}$.

It follows from [4] and [13] that $\mathcal{T} \mapsto \mathcal{T} \cap\{X\}^{\perp_{\geq 0}}$ defines a bijection between the torsion classes $\mathcal{T}$ in Mod- $H$ containing $X$ as an Ext-projective module, and the torsion classes in $\{X\}^{\perp \geq 0}$. Since $X$ has $n-1$ pairwise non-isomorphic indecomposable direct summands, the category $X^{\perp \geq 0}$ is equivalent to Mod- $K$, where $K$ is a $k$-division algebra; see Section 1 . Mod- $K$ only contains the trivial torsion classes $\{0\}$ and Mod- $K$. Hence $\operatorname{Gen}(X)$ and $\{X\}^{\perp}$ are the only torsion classes in Mod- $H$, containing $X$ as an Ext-projective module. The torsion class $\{X\}^{\perp}$ is the tilting torsion class of a finite dimensional tilting module $T^{\prime}$. If $X$ is not sincere, then $\operatorname{Gen}(X)$ is not a tilting torsion class since there are indecomposable injective $H$-modules not contained in $\operatorname{Gen}(X)$. Therefore $T \in \operatorname{Add}\left(T^{\prime}\right)$, and hence $T$ is equivalent to $T^{\prime}$ in this case. If $X$ is sincere, then $\operatorname{Gen}(X)$ also is the tilting torsion class of a finite dimensional tilting module $T^{\prime \prime}$; see [18]. Hence $T \in \operatorname{Add}\left(T^{\prime}\right)$ or $T \in \operatorname{Add}\left(T^{\prime \prime}\right)$ in the second case.

The proof of part (2) is by induction on $n$, but requires some preparation:

Proposition 2.4. Let $H$ be a representation-infinite connected hereditary artin algebra with $n$ simple modules. Then the following holds true:

(1) There exists a quasi-simple regular $H$-module $X$ with $\operatorname{Ext}_{H}^{1}(X, X)=0$, if and only if $n>2$.

(2) Let $n>2, X$ be quasi-simple regular without self-extensions, and $\{X\}^{\perp_{\geq 0}} \cong$ Mod- $C$ where $C$ is hereditary with $n-1$ simple modules. Then $C$ is connected to the same representation type (tame or wild) as $H$.

If $0 \rightarrow \tau_{H} X \rightarrow Z \rightarrow X \rightarrow 0$ is the Auslander-Reiten sequence ending in $X$, then $Z \in\{X\}^{\perp \geq 0}$ and $Z$ is quasi-simple regular in mod- $C$.

If $H$ is tame and $X$ belongs to a tube of rank $p>1$, then $Z$ belongs to a tube of rank $p-1$ in mod- $C$.

Proof. The first part is proved in [33], and the second in [34].

Let $H$ be a hereditary artin algebra and $X$ a finite dimensional $H$-module without self-extensions. Then $\{X\}^{\perp \geq 0} \cong \operatorname{Mod}-C$, where $C$ is a hereditary artin algebra. To simplify the notation, we will identify these categories.

For $Y \in\{X\}^{\perp \geq 0} \cap \bmod -H=\bmod -C$ with $\operatorname{Ext}_{C}^{1}(Y, Y)=0=\operatorname{Ext}_{H}^{1}(Y, X)$, we consider the full subcategory $\left\{Y_{C}\right\}^{\perp \geq 0}$ of Mod-C. This category then coincides with the full subcategory $\{X \oplus Y\}^{\perp_{\geq 0}}$ in Mod- $H$.

Lemma 2.5. Let $H$ be a wild connected hereditary artin algebra with $n>2$ simple modules, and $i$ be a natural number with $1 \leq i \leq n-2$. Then there exists a regular partial tilting module $X=\bigoplus_{j=1}^{i} X_{j}$, where the modules $X_{j}$ are indecomposable in mod- $H$, and for each $1 \leq j<i$, the following holds:

(1) There exists an epimorphism $\phi_{j}: X_{j+1} \rightarrow X_{j}$;

(2) $X_{j+1}$ is a quasi-simple regular module in $\left\{\bigoplus_{1 \leq a \leq j} X_{a}\right\}^{\perp_{\geq 0}}$. 
Proof. The proof is by induction on $i$. For $i=1$, we just choose a quasi-simple regular $H$-module $X_{1}$ without self-extensions; see Proposition 2.4(1).

Let $i>1$ and choose a quasi-simple regular $H$-module $X_{1}$ with $\operatorname{Ext}_{H}^{1}\left(X_{1}, X_{1}\right)=$ 0 . We identify $\left\{X_{1}\right\}^{\perp \geq 0}(\cong \operatorname{Mod}-C)$ with Mod-C. Then $C$ is a connected wild hereditary artin algebra with $n-1$ simple modules; see Proposition 2.4(2). By induction, we find a regular partial tilting module $Y=Y_{1} \oplus \cdots \oplus Y_{i-1}$ in mod- $C$ satisfying (1) and (2).

Let $0 \rightarrow \tau_{H} X_{1} \rightarrow Z_{1} \rightarrow X_{1} \rightarrow 0$ be the Auslander-Reiten sequence, ending in $X_{1}$. We know that $Z_{1}$ is a regular $C$-module. By $[20,28]$ there exists a natural number $m$ with the following properties:

(a) $0=\operatorname{Ext}_{C}^{1}\left(\tau_{C}^{-m} Y, Z_{1}\right)=\operatorname{Ext}_{H}^{1}\left(\tau_{C}^{-m} Y, Z_{1}\right)$.

(b) There is an epimorphism $\psi: \tau_{C}^{-m} Y_{1} \rightarrow Z_{1}$.

Since $\tau_{C}^{-m} Y \in\left\{X_{1}\right\}^{\perp \geq 0}$, we have $\operatorname{Ext}_{H}^{1}\left(X_{1}, \tau_{C}^{-m} Y\right)=0$. From the choice of $m$ we get $0=\operatorname{Ext}_{H}^{1}\left(\tau_{C}^{-m} Y, Z_{1}\right)=\operatorname{Ext}_{H}^{1}\left(\tau_{C}^{-m} Y, X_{1}\right)$. Hence $X=X_{1} \oplus \tau_{C}^{-m} Y$ is a partial tilting module. Defining $X_{j+1}=\tau_{C}^{-m} Y_{j}$, for $1 \leq j \leq i-1$, a straightforward computation yields the properties (1) and (2) for $X$.

Lemma 2.6. Let $H$ be a hereditary artin algebra and $X$ be a finitely generated indecomposable $H$-module without self-extensions. Let $\{X\}^{\perp \geq 0}=\operatorname{Mod}-C$, and $T^{\prime}$ be a tilting $C$-module which generates $X$. Then $T=T^{\prime} \oplus X$ is a tilting $H$-module.

Proof. Since $H$ is hereditary, p.dim $T \leq 1$. Since $X$ is finite dimensional, $\operatorname{Ext}_{H}^{1}(X, T)$ $=0$ implies $\operatorname{Ext}_{H}^{1}\left(X, T^{(I)}\right)=0$ for all sets $I$.

From $0=\operatorname{Ext}_{C}^{1}\left(T^{\prime}, T^{\prime(I)}\right) \cong \operatorname{Ext}_{H}^{1}\left(T^{\prime}, T^{\prime(I)}\right)$ we deduce $\operatorname{Ext}_{H}^{1}\left(T^{\prime}, X^{(I)}\right)=0$ for all sets $I$, since $X$ is generated by $T^{\prime}$; hence (T2) holds. We have to show that condition (T3) holds for $T$.

Let

$$
0 \rightarrow H \stackrel{u}{\rightarrow} P \stackrel{v}{\rightarrow} X^{r} \rightarrow 0
$$

be Bongartz's universal short exact sequence [10]. Since $X$ is indecomposable, $P$ is a projective generator in $\{X\}^{\perp_{\geq 0}}=\operatorname{Mod}-C$. Since $T^{\prime}$ is a tilting $C$-module, there exists a short exact sequence

$$
0 \rightarrow P \stackrel{f}{\rightarrow} T_{1} \stackrel{g}{\rightarrow} T_{2} \rightarrow 0
$$

with $T_{1}, T_{2} \in \operatorname{Add}\left(T^{\prime}\right)$.

Forming the pushout of $f$ and $v$, we get the following commutative diagram, with exact rows and columns:

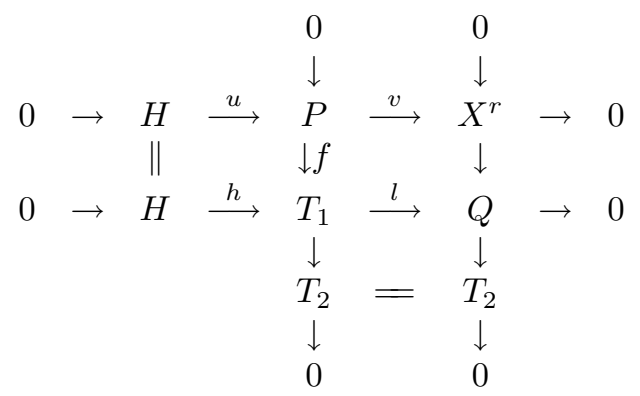

Since $\operatorname{Ext}_{H}^{1}\left(T_{2}, X\right)=0$, the last column splits, that is, $Q=T_{2} \oplus X^{r}$. So $T$ is a tilting $H$-module. 
Proof of Theorem A(2). For $i=0$, let $\mathcal{P}$ be the set of preprojective modules in mod- $H$. Then $\mathcal{P}^{\perp}$ is a tilting torsion class, generated by a countably generated tilting module $T_{\infty},[28,25]$. Since $\mathcal{T}_{\infty}=\mathcal{P}^{\perp} \cap$ ind- $H$ consists of the indecomposable regular and preinjective modules, there is no non-zero Ext-projective module in $\mathcal{T}_{\infty}$. Consequently $T_{0}=T_{\infty}$ has no indecomposable finite dimensional direct summand; see Lemma 2.1.

For $i>0$ first let $H$ be wild and consider the partial tilting module $X=X_{1} \oplus \cdots \oplus$ $X_{i}$ from Lemma 2.5. Let $\left\{\bigoplus_{1 \leq j \leq i-1} X_{j}\right\}^{\perp_{\geq 0}}=\operatorname{Mod}-C^{\prime}$. Then $X_{i}$ is a quasi-simple regular $C^{\prime}$-module, with Auslander-Reiten sequence $0 \rightarrow \tau_{C^{\prime}} X_{i} \rightarrow Z_{i} \rightarrow X_{i} \rightarrow 0$ in mod- $C^{\prime}$. Moreover we know that $Z_{i}$ is quasi-simple regular in $\{X\}^{\perp \geq 0}=\operatorname{Mod}-C^{\prime \prime}$. Choose in Mod- $C^{\prime \prime}$ the infinite dimensional tilting module (without indecomposable finite dimensional direct summands) $T_{\infty}^{\prime \prime}$, with $\left\{T_{\infty}^{\prime \prime}\right\}^{\perp}=\mathcal{P}\left(C^{\prime \prime}\right)^{\perp}$ as in the case of $i=0$. Since $Z_{i}$ is a regular $C^{\prime \prime}$-module, it is generated by $T_{\infty}^{\prime \prime}$. Hence there exists an epimorphism $\phi: T_{\infty}^{\prime \prime l} \rightarrow Z_{i} \rightarrow X_{i}$, for some $l>0$. Consequently we have a chain of epimorphisms

$$
T_{\infty}^{\prime \prime l} \rightarrow X_{i} \rightarrow \cdots \rightarrow X_{1}
$$

Let $T_{i}=T_{\infty}^{\prime \prime} \oplus X_{i} \oplus \cdots \oplus X_{1}$. It follows from Lemma 2.6 by induction that $T_{i}$ is a tilting $H$-module.

If $H$ is tame hereditary, then there exist $1 \leq r \leq 3$ inhomogeneous tubes $\mathcal{T}_{1}, \ldots, \mathcal{T}_{r}$ of rank $p_{1}, \ldots, p_{r}$ where $\sum\left(p_{i}-1\right)=n-2$. Let $Y_{s} \in \mathcal{T}_{s}$ be quasisimple and choose natural numbers $0 \leq i_{s}<p_{s}$ with $\sum_{s=1}^{r} i_{s}=i$. For each $s$ we consider the chain of irreducible epimorphism in $\mathcal{T}_{s}($ see $(* *))$

$$
\left(i_{s}+1\right) Y_{s} \rightarrow\left(i_{s}\right) Y_{s} \rightarrow \cdots \rightarrow(1) Y_{s}=Y_{s} .
$$

Let $Y_{(s)}=\bigoplus_{t=1}^{i_{s}}(t) Y_{s}$ and $X=\bigoplus Y_{(s)}$ Then $X$ is a partial tilting $H$-module with $i$ indecomposable direct summands. Let $\{X\}^{\perp \geq 0} \cong \operatorname{Mod}-C^{\prime \prime}$. Then $C^{\prime \prime}$ is a connected tame hereditary algebra with $n-i$ simple modules. Moreover, the modules $\left(i_{s}+1\right) Y_{s}$ are quasi-simple regular $C^{\prime \prime}$-modules, contained in tubes of rank $p_{s}-i_{s}$. In analogy to the first case, we chose the tilting $C^{\prime \prime}$-module $T_{\infty}^{\prime \prime}$ with $\left\{T_{\infty}^{\prime \prime}\right\}^{\perp}=\mathcal{P}\left(C^{\prime \prime}\right)^{\perp}$. Since $T_{\infty}^{\prime \prime}$ generates all the modules $\left(i_{s}+1\right) Y_{s}$, it generates $X$, hence again we get for $T_{i}=T_{\infty}^{\prime \prime} \oplus X$ that $\operatorname{Ext}_{H}^{1}\left(T_{i}, T_{i}^{(I)}\right)=0$, for all sets $I$. As in the wild case, Lemma 2.6 and induction give that $T_{i}$ is a tilting module in Mod- $H$.

Finally, we have to show in both cases that an indecomposable finite dimensional direct summand $Y$ of $T_{i}=T_{\infty}^{\prime \prime} \oplus X$ is isomorphic to some direct summand of $X$. Since $T_{\infty}^{\prime \prime}$ has no finite dimensional indecomposable direct summand, $Y$ is a direct summand of $X$ by Lemma 2.2. Therefore $Y$ is isomorphic to some indecomposable direct summand of $X$ by the Krull-Schmidt theorem.

\section{Direct summands of TILTing MOdules}

In this section, Theorem B will be proved. Most steps of the proof work in more generality: Recall, that for a class $\mathcal{C}$ of $R$-modules, a morphism $f: M \rightarrow C_{0}$ with $C_{0} \in \mathcal{C}$ is called a $\mathcal{C}$-preenvelope or also left $\mathcal{C}$-approximation, if for any $C \in \mathcal{C}$ the induced morphism $\operatorname{Hom}_{R}(f, C): \operatorname{Hom}_{R}\left(C_{0}, C\right) \rightarrow \operatorname{Hom}_{R}(M, C)$ is surjective. The preenvelope $f$ is called an envelope or minimal left approximation, if for any endomorphism $g$ of $C_{0}$ the condition $g f=f$ implies that $g$ is an automorphism. In this case the image of $f$ cannot be contained in a proper direct summand of $C_{0}$. 
Proposition 3.1. Let $R$ be a ring, and $M$ be an $R$-module. Then $M$ is a direct summand of a tilting module $T$ with $\operatorname{Gen}(T)=\operatorname{Gen}(M)$ if and only if $\operatorname{p.dim} M \leq 1$, $\operatorname{Ext}_{R}^{1}\left(M, M^{(I)}\right)=0$ for all sets $I, M$ is a faithful $R$-module, and $M$ is finitely generated over its endomorphism ring.

Proof. If there exists a tilting module $T=M \oplus Y$ with $\operatorname{Gen}(T)=\operatorname{Gen}(M)$, then clearly $\operatorname{Ext}_{R}^{1}\left(M, M^{(I)}\right)=0$ and p.dim $M \leq 1$ holds. Moreover, $\operatorname{Gen}(T)=\operatorname{Gen}(M)$ implies that $M$ is faithful and finendo by [13, Proposition 2.5].

Conversely, assume $M$ satisfies the four conditions of the proposition. Since $M$ is finendo, by [2, Proposition 1.2] $R$ has an add( $M)$-preenvelope $f: R \rightarrow P$. The map $f$ is necessarily monic, since $M$ is faithful. Hence it gives rise to a exact sequence

$$
0 \rightarrow R \stackrel{f}{\rightarrow} P \rightarrow Q \rightarrow 0
$$

Let $T=M \oplus Q$.

Clearly, $\operatorname{Gen}(T)=\operatorname{Gen}(M)$. Since p.dim $M \leq 1$, also p.dim $Q \leq 1$, and condition (T1) holds. The exact sequence $(\epsilon)$ yields condition (T3).

It remains to prove condition (T2). From $\operatorname{Ext}_{R}^{1}\left(M, M^{(I)}\right)=0$ and $(\epsilon)$ one gets $\operatorname{Ext}_{R}^{1}\left(M, T^{(I)}\right)=0$ for any set $I$. Since $p \cdot \operatorname{dim} Q \leq 1$, it remains to prove that $\operatorname{Ext}_{R}^{1}\left(Q, M^{(I)}\right)=0$ for any set $I$.

However, $f$ is an $\operatorname{add}(M)$ - and hence an $\operatorname{Add}(M)$-preenvelope, so the map $\operatorname{Hom}_{R}\left(f, M^{(I)}\right)$ is surjective, and we have the exact sequence

$$
\operatorname{Hom}_{R}\left(P, M^{(I)}\right) \stackrel{\operatorname{Hom}_{R}\left(f, M^{(I)}\right)}{\longrightarrow} \operatorname{Hom}_{R}\left(R, M^{(I)}\right) \rightarrow \operatorname{Ext}_{R}^{1}\left(Q, M^{(I)}\right) \rightarrow \operatorname{Ext}_{R}^{1}\left(P, M^{(I)}\right) .
$$

Since $\operatorname{Ext}_{R}^{1}\left(P, M^{(I)}\right)=0$, we conclude that $\operatorname{Ext}_{R}^{1}\left(Q, M^{(I)}\right)=0$.

In general, it is not easy to check whether a module (without self-extensions) is faithful. We will see that this becomes easier when the underlying ring is a right hereditary artinian ring, generalizing a well known result on finite dimensional modules over hereditary artin algebras; see for example $[23,8.3]$.

First, recall that a module $M$ over a ring $R$ is sincere if $\operatorname{Hom}_{R}(P, M) \neq 0$ for all non-zero projective modules $P$. Clearly, any faithful module is sincere. If $R$ is a right perfect ring, then a module $M$ is sincere if and only if all simple modules appear as subfactors of $M$.

Lemma 3.2. Let $H$ be a right hereditary right artinian ring and $M$ be a module with $\operatorname{Ext}_{H}^{1}(M, M)=0$. Then $M$ is faithful if and only if $M$ is sincere.

Proof. It suffices to show that $M$ is faithful provided $M$ is sincere. Let $L=$ $\operatorname{Ann}_{H}(M)$. Since $H$ is right artinian, the module $H / L$ is finitely cogenerated. So there is a finite subset $F \subseteq M$ such that $L=\bigcap_{m \in F} \operatorname{Ann}_{H}(m)$, and $L$ is the kernel of the map $f: H \rightarrow M^{F}$ defined by $f(h)=(m h)_{m \in F}$. Denote by $I$ the image of $f$, and consider the short exact sequence $0 \rightarrow L \hookrightarrow H \stackrel{p}{\longrightarrow} I \rightarrow 0$. By construction, the map $\operatorname{Hom}_{H}(p, M): \operatorname{Hom}_{H}(I, M) \rightarrow \operatorname{Hom}_{H}(H, M)$ is surjective, hence $\operatorname{Hom}_{H}(L, M) \cong \operatorname{Ext}_{H}^{1}(I, M)$. Since $I$ is a submodule of $M^{F}$ and $\operatorname{Ext}_{H}^{1}\left(M^{F}, M\right)=0$, we infer that $\operatorname{Hom}_{H}(L, M) \cong \operatorname{Ext}_{H}^{1}(I, M)=0$. Since $H$ is right hereditary, the module $L$ is projective. But $M$ is sincere, so $L=0$, and $M$ is faithful. 
Proposition 3.3. Let $R$ be a ring, $I^{2}=I$ an idempotent two-sided ideal of $R$, finitely generated as right ideal, and $\bar{T}$ a tilting $\bar{R}$-module where $\bar{R}=R / I$. Assume that $\mathrm{p} \cdot \operatorname{dim}_{R} \bar{T} \leq 1$.

Then $\bar{T}$ is a direct summand in a tilting module $T$ with $T^{\perp}=\bar{T}^{\perp}$.

Proof. First, since $I$ is idempotent, we have $\operatorname{Ext}_{\bar{R}}^{1}(A, B)=\operatorname{Ext}_{R}^{1}(A, B)$ for all $\bar{R}$ modules $A$ and $B$. In particular, $\operatorname{Ext}_{R}^{1}\left(\bar{T}, \bar{T}^{(X)}\right)=0$ for any set $X$.

Since $\operatorname{p\cdot dim} \operatorname{dim}_{R} \leq 1$, it remains only to prove that the class $\bar{T}^{\perp}$ is closed under arbitrary direct sums (then $\bar{T}$ is partial tilting, and $T$ is obtained by the Bongartz construction; cf. [13, Lemma 1.8]).

Assume that $\operatorname{Ext}_{R}^{1}\left(\bar{T}, M_{j}\right)=0$ for a family of $R$-modules $M_{j}(j \in J)$. Since $\bar{T}^{\perp} \cap \operatorname{Mod}-\bar{R}$ is a class of $\bar{R}$-modules of finite type [9, Theorem 2.4], there is a set, $\mathcal{S}$, of finitely presented $\bar{R}$-modules of projective dimension $\leq 1$ such that $\bar{R} \in \mathcal{S}$, and $\bar{T}^{\perp} \cap \operatorname{Mod}-\bar{R}=\mathcal{S}^{\perp} \cap \operatorname{Mod}-\bar{R}$.

By [35, Theorem 2.2], $\bar{T}$ is a direct summand in an $\bar{R}$-module, $T^{\prime}$, such that there are an ordinal $\lambda$ and an increasing chain, $\left(T_{\alpha} \mid \alpha<\lambda\right)$, consisting of $\bar{R}$ submodules of $T^{\prime}$ and satisfying $T_{0}=0, T_{\alpha}=\bigcup_{\beta<\alpha} T_{\beta}$ for each limit ordinal $\alpha<\lambda, T^{\prime}=\bigcup_{\alpha<\lambda} T_{\alpha}$, and $T_{\alpha+1} / T_{\alpha}$ is isomorphic to an element of $\mathcal{S}$ for each $\alpha+1<\lambda$. Similarly, by [35, Theorem 3.3], each $S \in \mathcal{S}$ is a direct summand in an $\bar{R}$-module $N_{S}$ such that $N_{S}$ is an extension of a free $\bar{R}$-module by a direct sum of copies of $\bar{T}$.

By assumption on $\bar{T}$, there is a short exact sequence $0 \rightarrow \bar{R} \rightarrow A \rightarrow B \rightarrow 0$ where $A, B \in \operatorname{Add}(\bar{T})$. So $\operatorname{Ext}_{R}^{1}\left(\bar{R}, M_{j}\right)=0$, hence $\operatorname{Ext}_{R}^{1}\left(N_{S}, M_{j}\right)=0$, and consequently $\operatorname{Ext}_{R}^{1}\left(S, M_{j}\right)=0$, for all $S \in \mathcal{S}$ and $j \in J$. Since $I$ is finitely generated as a right ideal, a finitely presented right $\bar{R}$-module remains finitely presented, when considered as an $R$-module. Since $S \in \mathcal{S}$ is finitely presented as an $R$-module, we have $\operatorname{Ext}_{R}^{1}\left(S, \bigoplus_{j \in J} M_{j}\right)=0$, and hence $\operatorname{Ext}_{R}^{1}\left(T^{\prime}, \bigoplus_{j \in J} M_{j}\right)=0$. Then also $\operatorname{Ext}_{R}^{1}\left(\bar{T}, \bigoplus_{j \in J} M_{j}\right)=0$.

The condition $I^{2}=I$ cannot be dropped in Proposition 3.3, since otherwise we can easily have $\operatorname{Ext}_{R}^{1}(\bar{T}, \bar{T}) \neq 0=\operatorname{Ext}_{\bar{R}}^{1}(\bar{T}, \bar{T})$ even if $\bar{T}$ is a free $\bar{R}$-module (just take $R=\mathbb{Z}$ and $I=\mathbb{Z} n$ for an integer $n>1$ ).

Proof of Theorem B. Let $X$ be as in Theorem B.

(a) Assume $X$ is sincere. Then $X$ is faithful by Lemma 3.2. By Proposition 3.1, there exists a tilting module $T=X \oplus Y$ with $\{T\}^{\perp}=\operatorname{Gen}(X)$.

(b) If $X$ is not sincere, let $H^{\prime}$ be the support ring of $X$, that is, let $H^{\prime}=$ $H /(H e H)$ where $e$ is the sum of all primitive idempotents $e_{i}$ with $X e_{i}=0$. Then $\mathrm{HeH}$ is an idempotent ideal in $H$, finitely generated as a right $H$-module. Since $\operatorname{Ext}_{H^{\prime}}^{1}(A, B)=\operatorname{Ext}_{H}^{1}(A, B)$ for all $H^{\prime}$-modules $A$ and $B$, the ring $H^{\prime}$ is a right hereditary and right artinian factor ring of $H$, and $X$ is a sincere $H^{\prime}$-module with $\operatorname{Ext}_{H^{\prime}}^{1}\left(X, X^{(I)}\right)=0$. So part (a) applies and we get a tilting $H^{\prime}$-module $T^{\prime}=X \oplus Y^{\prime}$. The tilting $H^{\prime}$-module $T^{\prime}$ can be completed to a tilting $H$-module $T=X \oplus Y^{\prime} \oplus Y^{\prime \prime}$ by Proposition 3.3.

Remark 3.4. If $X$ is a stone, then $E=\operatorname{End}_{H}(X)$ is a skew field (see [15]), and for each finite dimensional $H$-module $M$ the dimension $\operatorname{dim}_{E} \operatorname{Hom}_{H}(M, X)$ is finite. If $\left\{g_{1}, \ldots, g_{t}\right\}$ is an $E$-basis of $\operatorname{Hom}_{H}(M, X)$, the map $g=\left(g_{1}, \ldots, g_{t}\right): M \rightarrow X^{t}$ clearly is an $\operatorname{Add}(X)$-envelope of $M$. If moreover $X$ is sincere, hence faithful, and $\left\{f_{1}, \ldots, f_{r}\right\}$ is a basis of $\operatorname{Hom}_{H}(H, X)$, considered as an $E$-vector space, then the 
morphism $f=\left(f_{1}, \ldots, f_{r}\right): H \rightarrow X^{r}$ is the $\operatorname{Add}(X)$-envelope of $H$, and there is a tilting module $T=X \oplus Y$ induced by the short exact sequence

$$
0 \rightarrow H \stackrel{f}{\longrightarrow} X^{r} \longrightarrow Y \rightarrow 0 .
$$

It is easy to check that $f: H \rightarrow X^{r}$ is an $\operatorname{Add}(T)$-envelope, simultaneously.

If $P$ is a direct summand of $H$, say $H=P \oplus P^{\prime}$, by the Snake lemma we receive the following exact and commutative diagram:

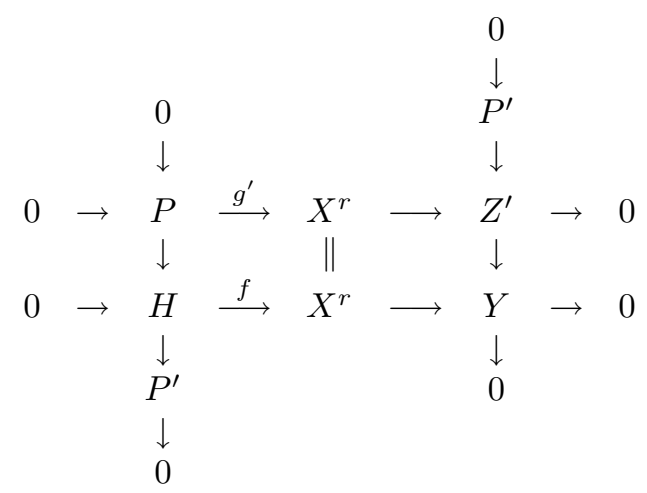

As in the case of finite dimensional tilting modules one checks that $Z^{\prime} \in \operatorname{Add}(T)$. Moreover, $g^{\prime}: P \rightarrow X^{r}$ is an $\operatorname{Add}(T)$ - and an $\operatorname{Add}(X)$-preenvelope, simultaneously. Since $P$ has an $\operatorname{Add}(X)$-envelope, the first row induces a short exact sequence

$$
0 \rightarrow P \stackrel{g}{\longrightarrow} X^{t} \rightarrow Z \rightarrow 0
$$

where $g: P \rightarrow X^{t}$, for some $t \leq r$, is an $\operatorname{Add}(X)$-envelope and $Z \in \operatorname{Add}(T)$.

In the case when $H$ is a tame connected hereditary algebra, $G$ is the generic module and $\operatorname{dim}_{E} G=r$, in the short exact sequence

$$
0 \rightarrow H \rightarrow G^{r} \rightarrow Y \rightarrow 0,
$$

the tilting module $T=G \oplus Y$ is equivalent to $G \oplus\left(\bigoplus_{\lambda \in k \cup \infty} R_{\lambda}\right)$ where $\left\{R_{\lambda}\right\}$ is the set of all Prüfer modules. For more details, we refer to [1].

\section{ITERATED ONE-POINT EXTENSIONS}

In some cases, the torsion class $\{T\}^{\perp}$ in Proposition 3.3 can be described rather precisely. Let $B$ be a finite dimensional $k$-algebra and $0 \neq R \in \bmod -B$. The one-point extension $A=B[R]$ of $B$ by $R$ is the generalized lower triangular matrix ring

$$
A=B[R]=\left(\begin{array}{ll}
B & 0 \\
R & k
\end{array}\right) .
$$

Then $e_{\omega}=\left(\begin{array}{ll}0 & 0 \\ 0 & 1\end{array}\right)$ is a primitive idempotent in $A$ and the indecomposable projective $A$-module $P_{\omega}=e_{\omega} A$ has radical $\left(\begin{array}{cc}0 & 0 \\ R & 0\end{array}\right)$. The injective $A$-module $S_{\omega}=\mathrm{D}\left(A e_{\omega}\right)$ is simple, and $\left(1_{A}-e_{\omega}\right) A\left(1_{A}-e_{\omega}\right) \cong B \cong A /\left(A e_{\omega} A\right)$.

Following [32], we will identify right $A$-modules $M$ with the triples $\left(M_{0}, V, \phi\right)$ where $M_{0} \in \operatorname{Mod}-B, V \in \operatorname{Mod}-k$ and $\phi: V \rightarrow \operatorname{Hom}_{B}\left(R, M_{0}\right)$ is a $k$-linear map. Also, we will identify Mod- $B$ with the full subcategory $\left\{\left(M_{0}, 0,0\right) \mid M_{0} \in \operatorname{Mod}-B\right\}$ 
of Mod- $A$. Consequently $B$ can be considered as a right ideal in $A$, and for a $B$-module $M_{0}$ we have p.dim ${ }_{B} M_{0}=$ p.dim ${ }_{A} M_{0}$.

For the global dimension of $A=B[R]$ one has g.d. $A=\max \{$ g.d. $B, 1+$ p.dim $R\}$. Consequently $A$ is hereditary, if and only if $B$ is hereditary and $R$ is projective.

For $M=\left(M_{0}, V, \phi\right) \in \operatorname{Mod}-A$ we have a short exact sequence

$$
\eta: \quad 0 \rightarrow M_{0} \rightarrow M \rightarrow S_{\omega}^{(\operatorname{dim} V)} \rightarrow 0
$$

in Mod- $A$. It is also shown in [32] that for an indecompososable module $X \in \bmod -B$ and for the Auslander-Reiten translations $\tau_{A}$, respectively $\tau_{B}$, one has

$$
\tau_{A} X=\left(\tau_{B} X, \operatorname{Hom}_{B}\left(R, \tau_{B} X\right), 1\right) .
$$

A finite dimensional $K$-algebra $A$ is called an iterated one-point extension (or, more precisely, an $m$-fold one-point extension) of $B$ if there is a sequence

$$
B=A_{0}, A_{1}, \ldots, A_{m}=A
$$

such that $A_{i}=A_{i-1}\left[R_{i-1}\right]$ is a one-point extension of $A_{i-1}$ by some module $R_{i-1} \in$ mod- $A_{i-1}$. In this case, for an $A$-module $M$ we again denote by $M_{0}=M B$ the biggest submodule of $M$, having support in (the right ideal) $B \cong A /\left(A\left(1_{A}-1_{B}\right) A\right.$ ).

Let $A$ be an iterated one-point extension of $B$ and $\bar{T}$ be a tilting $B$-module. Then p.dim ${ }_{A} \bar{T}=$ p.dim $\operatorname{dim}_{B} \bar{T} \leq 1$, hence Proposition 3.3 applies. Thus there exists an $A$-module $C$ such that $T=\bar{T} \oplus C$ is a tilting $A$-module with $\{T\}^{\perp}=\{\bar{T}\}^{\perp}$. The $B$-submodule $T_{0}$ of $T$ then is of the form $T_{0}=\bar{T} \oplus C_{0}$. The tilting $B$-module $\bar{T}$ induces a torsion pair $(\overline{\mathcal{T}}, \overline{\mathcal{F}})$ in $\bmod -B$. The torsion pair in $\bmod -A$, induced by $T$, is denoted by $(\mathcal{T}, \mathcal{F})$.

Proposition 4.1. Let $A$ be an iterated one-point extension of some finite dimensional $k$-algebra $B$. Let $\bar{T}$ be a tilting $B$-module and $T=\bar{T} \oplus C$ a tilting A-module with $\{\bar{T}\}^{\perp}=\{T\}^{\perp}$. Then the following hold:

(1) $\{\bar{T}\}^{\perp}=\left\{M \in \operatorname{Mod}-A \mid M_{0} \in \operatorname{Gen}(\bar{T}) \subset \operatorname{Mod}-B\right\}$.

(2) $\mathcal{T}=\left\{M \in \bmod -A \mid M_{0} \in \overline{\mathcal{T}}\right\}$.

For $A$ hereditary, one has moreover:

(3) $\operatorname{Add}(\bar{T})=\operatorname{Add}\left(T_{0}\right)$.

(4) $\overline{\mathcal{F}}=\mathcal{F} \cap \bmod -B$.

(5) $T$ contains indecomposable finite dimensional direct summands if and only if $\bar{T}$ does.

Proof. By induction it is enough to consider the case where $A=B[R]$ is a one-point extension of $B$. Recall that for an $A$-module $M=\left(M_{0}, V, \phi\right)$, there is the short exact sequence

$$
\eta: \quad 0 \rightarrow M_{0} \rightarrow M \rightarrow S_{\omega}^{(I)} \rightarrow 0
$$

where $I=\operatorname{dim} V$ and $S_{\omega}$ is injective.

(1) We apply the functor $\operatorname{Hom}_{A}(\bar{T},-)$ to $\eta$ and get $0=\operatorname{Hom}_{A}\left(\bar{T}, S_{\omega}^{(I)}\right) \rightarrow$ $\operatorname{Ext}_{A}^{1}\left(\bar{T}, M_{0}\right) \rightarrow \operatorname{Ext}_{A}^{1}(\bar{T}, M) \rightarrow \operatorname{Ext}_{A}^{1}\left(\bar{T}, S_{\omega}^{(I)}\right)=0$. Hence $\left.M \in\{\bar{T})\right\}^{\perp}$ if and only if $M_{0} \in\{\bar{T}\}^{\perp}$ if and only if $M_{0} \in \operatorname{Gen}(\bar{T})$.

(2) follows from (1), since $\operatorname{Gen}(\bar{T}) \cap \bmod -B=\overline{\mathcal{T}}$.

For the remaining parts of the proof, assume that $A$ is hereditary.

(3) Consider the short exact sequence

$$
\rho: \quad 0 \rightarrow T_{0} \rightarrow T \rightarrow S_{\omega}^{(J)} \rightarrow 0 .
$$


For any set $L, \operatorname{Ext}_{A}^{1}\left(T, T^{(L)}\right)=0$, so $\operatorname{Ext}_{A}^{1}\left(T_{0}, T^{(L)}\right)=0$. Since $\operatorname{Hom}_{A}\left(T_{0}, S_{\omega}\right)=$ 0 , we get $0 \rightarrow \operatorname{Ext}_{A}^{1}\left(T_{0}, T_{0}^{(L)}\right) \rightarrow \operatorname{Ext}_{A}^{1}\left(T_{0}, T^{(L)}\right)=0$, hence $\operatorname{Ext}_{B}^{1}\left(T_{0}, T_{0}^{(L)}\right)=$ $\operatorname{Ext}_{A}^{1}\left(T_{0}, T_{0}^{(L)}\right)=0$. But $\bar{T}$ is a tilting $B$-module, and $T_{0}=\bar{T} \oplus C_{0}$. Since $B$ is hereditary, p.dim $T_{0} \leq 1$. Since $C_{0} \in \operatorname{Gen}(\bar{T})$, there exists a short exact sequence $0 \rightarrow L \longrightarrow \bar{T}^{(J)} \stackrel{\pi}{\longrightarrow} C_{0}$, with $L \in \operatorname{Gen}(\bar{T})$; see [13, Lemma 1.2.]. Since $C_{0}$ has projective dimension at most one and $\operatorname{Ext}_{B}^{1}\left(C_{0}, \bar{T}^{(J)}\right)=0$, the module $C_{0}$ is Extprojective in $\operatorname{Gen}(\bar{T})$, hence $\pi$ is a split epimorphism. Therefore $\operatorname{Add}\left(T_{0}\right)=\operatorname{Add}(\bar{T})$ holds.

(4) Since $\mathcal{F} \cap \bmod -B=\left\{M \in \bmod -B \mid \operatorname{Hom}_{A}(T, M)=0\right\}$, we get $\overline{\mathcal{F}} \supseteq \mathcal{F} \cap$ $\bmod -B$. Let $M \in \overline{\mathcal{F}}$. Since $T_{0} \in \operatorname{Add}(\bar{T})$, we get $\operatorname{Hom}_{A}\left(T_{0}, M\right)=0$. An application of $\operatorname{Hom}_{A}(-, M)$ to the short exact sequence $\rho$ then gives $0=\operatorname{Hom}_{A}\left(S_{\omega}^{(J)}, M\right) \rightarrow$ $\operatorname{Hom}_{A}(T, M) \rightarrow \operatorname{Hom}_{A}\left(T_{0}, M\right)$, hence $\operatorname{Hom}_{A}(T, M)=0$, which means that $M \in$ $\mathcal{F} \cap \bmod -B$.

(5) Since $\bar{T}$ is a direct summand of $T$, it is enough to show that if $T$ has an indecomposable finite dimensional direct summand $X$, then so does $\bar{T}$. Let $T=$ $X \oplus T^{\prime}$. Since $\operatorname{Ext}_{A}^{1}\left(S_{\omega}, \bar{T}\right) \neq 0$, we get $X \not S_{\omega}$. Therefore the submodule $X_{0}$ of $X$ is non-zero. We have $T_{0}=X_{0} \oplus T_{0}^{\prime} \in \operatorname{Add}(\bar{T})$, hence $0 \neq X_{0} \in \operatorname{add}(\bar{T})$. By Proposition 2.3 each indecomposable direct summand of $X_{0}$ is therefore a direct summand in $\bar{T}$.

If $A$ is not hereditary, part (3) and (5) of the proposition no longer hold:

Example 4.2. Let $B$ be connected wild hereditary, and $\bar{T}$ the tilting $B$-module without non-zero finite dimensionsional direct summands, such that $\overline{\mathcal{T}}$ consists of the direct sums of regular and preinjective $B$-modules. Let $R \neq 0$ be a regular $B$-module. The one-point extension $A=B[R]$ has global dimension two. The new projective $B[R]$-module $P_{\omega}$ is in $\mathcal{T}$, since so are $R$ and $S_{\omega}$. Therefore it is Ext-projective in $\mathcal{T}$ and consequently a direct summand of $T$. It is easy to see that $T$ is equivalent to $P_{\omega} \oplus \bar{T}$, hence (3) and (5) fail. Nevertheless we get (4) in this example since $\mathcal{F}=\mathcal{F} \cap \bmod -B=\overline{\mathcal{F}}$.

If $A=B[R]$ is a one-point extension and $\bar{T}$ is a finite dimensional tilting $B$ module, then there exists (a unique) indecomposable $A$-module $X_{0}$, such that $T=$ $\bar{T} \oplus X_{0}$ is a tilting $A$-module; see [18]. If $A$ additionally is hereditary, which means $B$ is hereditary and $R$ is a projective $B$-module, one can prove more.

Proposition 4.3. Let $A=B[R]$ be a hereditary $k$-algebra, and $\bar{T}$ be a tilting $B$-module. If $R$ admits an $\operatorname{Add}(\bar{T})$-envelope, then there exists an indecomposble $A$-module $X_{0}$, such that $T=\bar{T} \oplus X_{0}$ is a tilting $A$-module with $\{T\}^{\perp}=\{\bar{T}\}^{\perp}$.

Proof. Since $R$ is a projective $B$-module, there exists a short exact sequence

$$
0 \rightarrow R \stackrel{f}{\longrightarrow} \bar{T}_{1} \stackrel{g}{\longrightarrow} \bar{T}_{2} \rightarrow 0
$$

with $\bar{T}_{1}, \bar{T}_{2} \in \operatorname{Add}(\bar{T})$. The morphism $f$ clearly is an $\operatorname{Add}(\bar{T})$-preenvelope. Since $R$ admits an $\operatorname{Add}(\bar{T})$-envelope, we may assume that $f$ is already an $\operatorname{Add}(\bar{T})$-envelope; see [13]. Let $P_{\omega}$ be the indecomposable projective $A$-module with radical $R$, let $i: R \rightarrow P_{\omega}$ be the inclusion and let $S_{\omega}$ be the new simple $A$-module, which is injective. Forming the pushout of $f$ and $i$, we get the following exact and commutative 
diagram:

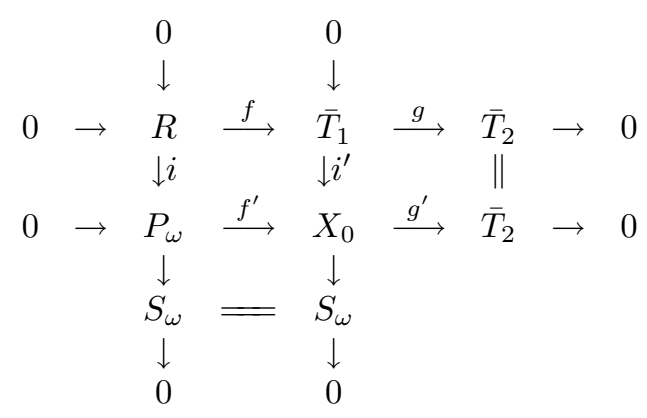

and we consider $i^{\prime}$ to be the inclusion. Since $\operatorname{Hom}_{A}\left(\bar{T}_{1}, S_{\omega}\right)=0$, we get $k \cong$ $\operatorname{Hom}_{A}\left(S_{\omega}, S_{\omega}\right) \cong \operatorname{Hom}_{A}\left(X_{0}, S_{\omega}\right)$. Let $X_{0}=U \oplus V$ with $\operatorname{Hom}_{A}\left(U, S_{\omega}\right) \neq 0$. Then $0=\operatorname{Hom}_{A}\left(V, S_{\omega}\right) \cong \operatorname{Hom}_{A}\left(P_{\omega}, V\right)$, since $S_{\omega}$ is injective. Therefore $f^{\prime}\left(P_{\omega}\right) \subset U$, hence we get $\bar{T}_{2} \cong\left(U / f^{\prime}\left(P_{\omega}\right)\right) \oplus V$. From $\operatorname{Hom}_{A}\left(V, S_{\omega}\right)=0$ we deduce $\bar{T}_{1}=i^{\prime}\left(\bar{T}_{1}\right)=$ $U^{\prime} \oplus V$, with $U^{\prime} \subset U$ and additionally $f(R) \subset U^{\prime}$. But $f: R \rightarrow \bar{T}_{1}$ is an $\operatorname{Add}(\bar{T})$ envelope, consequently $V=0$ follows. This implies that $X_{0}$ is indecomposable.

Let $I$ be any set. Since Mod- $B$ is closed under extensions in Mod- $A$, we get $0=$ $\operatorname{Ext}_{B}^{1}\left(\bar{T}, \bar{T}^{(I)}\right)=\operatorname{Ext}_{A}^{1}\left(\bar{T}, \bar{T}^{(I)}\right)$. From the second column of the diagram we infer $\operatorname{Ext}_{A}^{1}\left(\bar{T}, X_{0}^{(I)}\right)=0$, since $S_{\omega}$ is injective. The second row shows $\operatorname{Ext}_{A}^{1}\left(X_{0}, \bar{T}^{(I)}\right)=0$ and $\operatorname{Ext}_{A}^{1}\left(X_{0}, X_{0}^{(I)}\right)=0$. Hence $\operatorname{Ext}_{A}^{1}\left(T, T^{(I)}\right)=0$ holds for $T=\bar{T} \oplus X_{0}$. Since $\bar{T}$ is a tilting $B$-module, there exists a short exact sequence $0 \rightarrow B \rightarrow \bar{T}^{\prime} \rightarrow \bar{T}^{\prime \prime} \rightarrow 0$, with $\bar{T}^{\prime}, \bar{T}^{\prime \prime} \in \operatorname{Add}(\bar{T})$. Since $A=B \oplus P_{\omega}$, the short exact sequence

$$
0 \rightarrow A \rightarrow \bar{T}^{\prime} \oplus X_{0} \rightarrow \bar{T}^{\prime \prime} \oplus \bar{T}_{2} \rightarrow 0
$$

shows (T3).

The second row of the diagram also implies $\{\bar{T}\}^{\perp} \subset\left\{\bar{T}_{2}\right\}^{\perp} \subset\left\{X_{0}\right\}^{\perp}$, hence $\{T\}^{\perp}=\{\bar{T}\}^{\perp}$ follows.

If $H$ is a connected wild hereditary artin algebra, then an indecomposable regular module $E$ is called elementary, if there is no short exact sequence $0 \rightarrow U \rightarrow E \rightarrow$ $V \rightarrow 0$, where both $U$ and $V$ are non-zero and regular. Elementary modules always are quasi-simple with a trivial endomorphism ring, and $E$ is elementary if and only if $\tau_{H}^{i} E$ is elementary for all integers $i$. Moreover, each non-zero regular $H$-module $M$ has a filtration with elementary subquotients. Fore details see [29, 24].

Before presenting our final example, we collect some facts on tilting torsion pairs in mod- $H$ for a finite dimensional hereditary algebra $H$; see $[21,4]$.

Proposition 4.4. Let $H$ be a connected finite dimensional hereditary algebra, $(\mathcal{T}, \mathcal{F})$ a tilting torsion pair in mod- $H$, and $\mathcal{E}=\left\{T_{1}, \ldots, T_{r}\right\}$ a complete set of indecomposable Ext-projective modules in $\mathcal{T}$. Then the following hold:

(1) Let $X$ be an indecomposable non-projective torsion module. Then $\tau_{H} X \in \mathcal{T}$, if and only if $\operatorname{Hom}_{H}\left(X, T_{i}\right)=0$ for all $i$. If $\tau_{H} X$ is not torsion, then the add $(\mathcal{E})$-envelope $X \rightarrow \bigoplus_{i=1}^{r} T_{i}^{s_{i}}$ is surjective.

(2) Let $Y$ be an indecomposable non-injective torsion-free module. Then $\tau_{H}^{-} Y \in$ $\mathcal{F}$, if and only if $\operatorname{Hom}_{H}\left(\tau_{H} T_{i}, Y\right)=0$ for all $i$. If $\tau_{H}^{-} Y$ is not torsion-free, then the $\operatorname{add}\left(\tau_{H} \mathcal{E}\right)$-cover $\bigoplus_{i=1}^{r} \tau_{H} T_{i}^{t_{i}} \rightarrow Y$ is injective.

(3) If there exists a preprojective module which is not torsion-free, then there exists an indecomposable preprojective Ext-projective torsion module. 
(4) Let $H$ be wild hereditary and $R$ an indecomposable regular $H$-module. If $\tau_{H}^{i} R$ is torsion (respectively, torsion-free) for all integers $i$, then all regular and preinjective modules are torsion (all regular and preprojective modules are torsion-free, respectively).

(5) Let $H$ be wild hereditary and assume that all preprojective modules are torsion-free and all preinjective modules are torsion. Then each elementary module $E$ is either torsion or torsion-free.

Example 4.5. Let $K$ be an algebraically closed field and $H=K \mathcal{Q}$ be the path algebra of the quiver

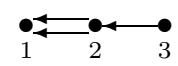

Then $H$ is a connected wild hereditary algebra with three simple modules. Also, $H$ is a one-point extension of the Kronecker algebra $A=H /\left(H_{3} H\right)$ by the projective module $P_{2}$, so Proposition 4.1 applies in this setting.

By [22], for regular $H$-modules $X$ and $Y$ with $\operatorname{Hom}_{H}(X, Y) \neq 0$, for all $i \geq 2$ we get $\operatorname{Hom}_{H}\left(X, \tau_{H}^{i} Y\right) \neq 0$, since $H$ has three simple modules. The elementary $H$ modules form the $\tau$-orbit of the indecomposable module $X$ with dimension vector $\underline{\operatorname{dim}} X=(5,4,0)$, and the $\tau$-orbits of the $P(1, K)$-family of indecomposable modules $Y_{\rho}, \rho \in P(1, K)$ with $\underline{\operatorname{dim}} Y_{\rho}=(1,1,0)$, by [29].

(1) Let $X$ be the elementary module with $\underline{\operatorname{dim}} X=(5,4,0)$. In $\{X\}^{\perp \geq 0}=$ Mod- $C$ we consider the tilting module $T_{\infty}$, defined by $\left\{T_{\infty}\right\}^{\perp}=\mathcal{P}(C)^{\perp}$; see $[28,25]$. This tilting $C$-module generates all regular and preinjective $C$-modules. Let $0 \rightarrow$ $\tau_{H} X \rightarrow(2) X \rightarrow X \rightarrow 0$ be the Auslander-Reiten sequence, ending in $X$. Then (2) $X$ is a regular $C$-module, hence it is generated by $T_{\infty}$. Consequently $X$ is also generated by $T_{\infty}$, hence $T=T_{\infty} \oplus X$ is a tilting $H$-module, by Lemma 2.6. Let $\mathcal{T}=T^{\perp} \cap$ ind- $H$. By construction $X$ is the only indecomposable Ext-projective module in $\mathcal{T}$, since $T_{\infty}$ has no non-zero finite dimensional direct summands; see Lemma 2.2.

Consequently all indecomposable preinjective $H$-modules are contained in $\mathcal{T}$, whereas all preprojective $H$-modules are torsion-free. Since $\operatorname{Hom}_{H}\left(X, \tau_{H}^{i} X\right) \neq 0$ for all $i>1$, the elementary modules $\tau_{H}^{i} X \in \mathcal{T}$, for $i>1$. Dually, all the modules $\tau_{H}^{-i} X$, for $i<0$, are torsion-free, since $\operatorname{Hom}_{H}\left(\tau_{H}^{-i} X, \tau_{H} X\right) \neq 0$. Similarly, from $\operatorname{Hom}_{H}\left(X, \tau_{H}^{i} Y_{\rho}\right) \neq 0$ for $i \geq 0$, we conclude $\tau_{H}^{i} Y_{\rho} \in \mathcal{T}$, for all $i \geq 0$ and all $\rho \in P(1, K)$. The modules $\tau_{H}^{i} Y_{\rho}$, for $i<0$, are torsion-free, since they map nontrivially to $\tau_{H} X$. Since each indecomposable regular module has a filtration with elementary subquotients, we conclude that each regular component $\mathcal{C}$ contains a quasi-simple module $U$, such that the cone $(\rightarrow U)$ of predecessors of $U$ in the component $\mathcal{C}$ consists of torsion modules. Here, $V$ is a predecessor of $U$ in $\mathcal{C}$ if there exists a path of arrows in the AR-quiver of $H$ of length $\geq 0$ from $V$ to $U$. Similarly, there exists a quasi-simple module $V=\tau_{H}^{-r} U$ for some $r>0$, such that all modules in the cone $(V \rightarrow)$ of successors of $V$ in $\mathcal{C}$ are torsion-free. Since $(2) X$ is generated by $T_{\infty}$, we get $(2) X \in \mathcal{T}$. Since the modules $\tau_{H}^{2}(j) X \in \mathcal{T}$, for all $j \geq 1$ and we have short exact sequences $\tau_{H}^{2}(i-2) X \rightarrow(i) X \rightarrow(i-1) X \rightarrow 0$, for all $i>0$, we conclude from $X,(2) X \in \mathcal{T}$ by induction that $(i) X \in \mathcal{T}$ for all $i$.

All regular $C$-modules are generated by $T_{\infty}$, hence they are torsion modules. If $R$ is an indecomposable regular $C$-module, then $\operatorname{Hom}_{C}\left(\tau_{C}^{-m} R,(2) X\right) \neq 0$, for $m \gg 0$, by [8]. Since $0 \neq \operatorname{Hom}_{C}\left(\tau_{C}^{-m} R,(2) X\right) \cong \operatorname{Hom}_{H}\left(\tau_{C}^{-m} R, X\right)$, we conclude 
from Proposition 4.4 that $\tau_{H} \tau_{C}^{-m} R \notin \mathcal{T}$. Hence there exist infinitely many indecomposable regular torsion modules $\tau_{C}^{-m} R$, not contained in the torsion cones $(\rightarrow U)$, described above; we call them the isolated torsion modules. By definition they are those indecomposable torsion modules $M$, such that $\tau_{H}^{r} M \notin \mathcal{T}$, for some $r>0$.

We first show that each regular component $\mathcal{C}$ contains at most one isolated quasi-simple torsion module. Indeed, suppose $U$ and $V=\tau_{H}^{-t} U$, with $t>0$, are quasi-simple isolated torsion modules in $\mathcal{C}$. We may assume that $\tau_{H} U \notin \mathcal{T}$, hence by Proposition $4.4 \operatorname{Hom}_{H}(U, X) \neq 0$. Consequently $\operatorname{Hom}_{H}\left(V, \tau_{H}^{-t} X\right) \neq 0$, but $\tau_{H}^{-t} X$ is torsion-free, for $i>0$, a contradiction.

Next we show that all isolated torsion modules, with the exception of the modules (i) $X$, are quasi-simple. Indeed, assume $M \nsubseteq(r) X$ is an indecomposable isolated torsion module of quasi-length $r>1$. Then there exists a quasi-simple regular module $S$ and a chain of irreducible epimorphisms

$$
M=(r) S \rightarrow(r-1) S \rightarrow \cdots \rightarrow(2) S \rightarrow S .
$$

Therefore all the modules $(i) S$, for $1 \leq i \leq r$, are indecomposable torsion modules. Consider the Auslander-Reiten sequence $0 \rightarrow \tau_{H} S \stackrel{f}{\longrightarrow}(2) S \stackrel{g}{\longrightarrow} S \rightarrow 0$. Since $M$ is an isolated torsion module, so is $S$. Therefore $S$ is the only quasi-simple isolated torsion module in $\mathcal{C}$, which implies $\tau_{H} S$ is not torsion. Since $S \neq X$, the module $\tau_{H} S$ is not torsion-free. Let $0 \rightarrow t\left(\tau_{H} S\right) \rightarrow \tau_{H} S \rightarrow f\left(\tau_{H} S\right) \rightarrow 0$ be the canonical short exact sequence, where $t\left(\tau_{H} S\right)$ is the torsion submodule of $\tau_{H} S$ and $f\left(\tau_{H} S\right)$ is torsion-free. Since the epimorphism $\tau_{H} S \rightarrow f\left(\tau_{H} S\right)$ is not a split monomorphism, it factorises through the source map $f: \tau_{H} S \rightarrow(2) S$. But (2) $S$ is a torsion module, a contradiction.

Dually, we call an indecomposable regular torsion-free module $F$, isolated torsionfree, if $\tau_{H}^{-r} F$ is not torsion-free, for some $r>0$. We now show that $\tau_{H} X$ is the only isolated torsion-free module. Indeed, suppose $M \nsubseteq \tau_{H} X$ is isolated torsion-free and $\tau_{H}^{-} M$ is not torsion-free By Proposition 4.4 there exists a non-zero morphism $h: \tau_{H} X \rightarrow M$, which clearly is not a split mono. Hence it factorises through the source map $f: \tau_{H} X \rightarrow(2) X$, but (2) $X$ is a torsion module, a contradiction.

(2) Let $S_{3}$ be the simple injective $H$-module, corresponding to the vertex 3 of the quiver. Let $\mathcal{A}=S_{3}^{\perp \geq 0} \subset \operatorname{Mod}-H$. Then $\mathcal{A}$ is equivalent to $\operatorname{Mod}-A$, where $A$ is the Kronecker-algebra, the minimal projective generator of $\mathcal{A}$ is the direct sum $P_{1} \oplus P_{3}$ of projective modules, and an $H$-module $M$ is in $\mathcal{A}$ if and only if $\operatorname{Hom}_{H}\left(M, S_{2}\right)=0$.

The equivalence $F: \operatorname{Mod}-A \rightarrow \mathcal{A}$ is given (in term of representations) by

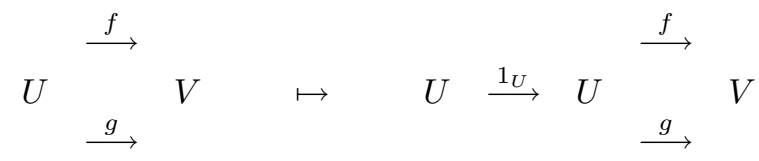

Let $T^{\prime}=G \oplus \bigoplus_{\lambda} R_{\lambda}$ be the tilting $A$-module, where $G$ is the generic $A$-module and $\left\{R_{\lambda} \mid \lambda \in P(1, K)\right\}$ the set of all Prüfer $A$-modules; see [1, Example 1.4]. Then $T^{\perp} \cap \bmod -A$ consists of the preinjective $A$-modules. Let $T_{1} \in \mathcal{A}$ be the image of $T^{\prime}$ under the equivalence $F$. The indecomposable injective module $I_{2}$ is preinjective in $\mathcal{A}$. Indeed, it is the simple injective object in $\mathcal{A}$, hence it is generated by $T_{1}$. Consequently $S_{3}$ is also generated by $T_{1}$. By Lemma $2.6 \hat{T}=T_{1} \oplus S_{3}$ is an $H$-tilting module with exactly one finite dimensional indecomposable direct 
summand $S_{3}$. Additionally, we know from the construction of $\hat{T}$ that $\{\hat{T}\}^{\perp}=$ $\operatorname{Gen}(F(G))$. It is easy to check that $\mathcal{T}=\hat{T}^{\perp} \cap$ ind- $H$ consists of the module $S_{3}$ and the countably many indecomposable $H$-modules $Z_{i}$ without self-extensions, where $\underline{\operatorname{dim}} Z_{i}=(i, i+1, i+1)$, for $i \geq 0$. Indeed, for $Z \in \mathcal{T}$ with $Z \not S_{3}$, we get $\operatorname{Hom}_{H}\left(S_{3}, Z\right)=0$ which means that $Z \in \mathcal{T} \cap \mathcal{A}$. Note that the modules $Z_{i}$ are regular for $i \geq 2$, that $Z_{2}$ is elementary, and that $Z_{0}$ and $Z_{1}$ are injective.

Since $S_{2}=\tau_{H} S_{3}$, the simple module $S_{2}$ is torsion-free [19]. If $I \neq S_{2}$ is an indecomposable preinjective module, then $\operatorname{Hom}_{H}\left(Z_{5}, I\right) \neq 0$, hence $S_{2}$ is the only indecomposable preinjective torsion-free module. Since $S_{3}$ is the unique indecomposable Ext-projective module in $\mathcal{T}$, all preprojective $H$-modules are torsion-free. The elementary modules $X$ with $\underline{\operatorname{dim}} X=(5,4,0)$ and the elementary modules $Y_{\lambda}$ with $\underline{\operatorname{dim}} Y_{\lambda}=(1,1,0)$ have filtrations with composition factors $S_{1}$ and $S_{2}$, therefore they are torsion-free. If $R$ is an indecomposable regular torsion-free module, then $\tau_{H}^{-r} R$ is torsion-free by Proposition 4.4, for all $r \geq 0$, since $\operatorname{Hom}_{H}\left(S_{2}, R^{\prime}\right)=0$ for all regular modules $R^{\prime}$, which means that there are no isolated regular torsion-free modules. Consequently all elementary modules $\tau_{H}^{-r} Y_{\lambda}$ and $\tau_{H}^{-r} X$ are torsion-free. Again we conclude that each regular component $\mathcal{C}$ contains a quasi-simple module $U$, such that the cone $(U \rightarrow)$ consists of the indecomposable torsion-free modules in $\mathcal{C}$. All torsion modules are isolated.

This example has another interpretation: We now consider the stone $X$, corresponding to the imaginary Schur root $(1,1,1)$. Then $X$ is the image of the generic $A$-module $G$ under the functor $F: \operatorname{Mod}-A \rightarrow \mathcal{A}$. Therefore $X$ is the representation

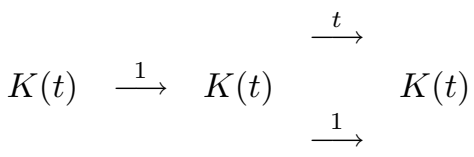

and $E=\operatorname{End}_{H}(X)=K(t)$, the rational function field. Since $X$ is sincere, hence faithful, we get by Proposition 3.1 a tilting module $T=X \oplus Y$ with $\{T\}^{\perp}=$ $\operatorname{Gen}(X)$. Since also $\{\hat{T}\}^{\perp}=\operatorname{Gen}(X)$, the tilting modules $T$ and $\hat{T}$ are equivalent.

(3) Let $A=H /\left(H_{3} H\right)$ be the Kronecker algebra. We identify Mod- $A$, respectively $\bmod -A$, with the full subcatecories of Mod- $H$, respectively mod- $H$, consisting of $H$-modules, having support in the full subquiver $\overline{\mathcal{Q}}$, defined by the vertices 1 and 2. In Mod- $A$ we consider the tilting $A$-module $T^{\prime}=G \oplus \oplus R_{\lambda}$, where $G$ is the generic module and $\left\{R_{\lambda}\right\}$ is the set of all Prüfer $A$-modules. Then $T^{\prime \perp} \cap \bmod -A$ consists of the preinjective $A$-modules. Let $T$ be a tilting $H$-module with $\{T\}^{\perp}=\left\{T^{\prime}\right\}^{\perp}$. Then we infer from Proposition 4.1:

(1) $M \in\{T\}^{\perp}$ if an only if $M_{0}=M A \in \operatorname{Gen}\left(T^{\prime}\right)$. If additionally $M$ is finite dimensional, then $M \in\{T\}^{\perp}$ if and only if $M_{0}$ is a preinjective $A$-module.

(2) $T$ has no finite dimensional indecomposable direct summands.

Let $(\mathcal{T}, \mathcal{F})$ be the torsion pair in mod- $H$, induced by $T$. From Lemma 2.1 and Proposition 4.4 we therefore deduce:

(a) $\tau_{H} \mathcal{T} \subset \mathcal{T}$ and $\tau_{H}^{-} \mathcal{F} \subset \mathcal{F}$.

(b) All preprojective modules are torsion free, and all preinjective modules are torsion.

The elementary $H$-module $X^{\prime}$ with $\underline{\operatorname{dim}} X=(1,2,0)$ is in $\mathcal{T}$, hence $\tau_{H}^{r} X^{\prime} \in \mathcal{T}$, for all $r \geq 0$. The elementary $H$-modules $Y_{\lambda}$ with $\underline{\operatorname{dim}} Y_{\lambda}=(1,1,0)$ are not torsion, but the elementary modules $\tau_{H}^{2} Y_{\lambda}$ have the biggest $A$-submodule $X^{\prime}$, hence they 
are torsion modules. Consequently all the modules $\tau_{H}^{r} Y_{\lambda}$, for $r \geq 2$ are torsion modules.

Again we conclude that each regular component $\mathcal{C}$ contains quasi-simple modules $U$ and $V=\tau_{H}^{-r} U$, for some $r>0$, such that the cone $(\rightarrow U)$ consists of torsion modules in $\mathcal{C}$ and the cone $(V \rightarrow)$ of the torsion free modules in $\mathcal{C}$.

We know from Remark 3.4 that the projective $A$-module $P_{2}$ admits an $\operatorname{Add}\left(T^{\prime}\right)$ envelope of the form

$$
0 \rightarrow P_{2} \rightarrow G \rightarrow T_{1}^{\prime} \rightarrow 0
$$

with $T_{1}^{\prime} \in \operatorname{Add}\left(T^{\prime}\right)$, since $\operatorname{dim}_{E} \operatorname{Hom}_{A}\left(P_{2}, G\right)=1$, for $E=\operatorname{End}_{A}(G) \cong k(t)$. Consequently, the tilting module $T$ with $\{T\}^{\perp}=\left\{T^{\prime}\right\}^{\perp}$ can be chosen by Proposition 4.3 as $T=T^{\prime} \oplus X_{0}$, with $X_{0}$ indecomposable. Indeed, $X_{0}$ is the middle term of the non-split short exact sequence $0 \rightarrow G \rightarrow X_{0} \rightarrow S_{3} \rightarrow 0$. Additionally, a direct calculation shows $\operatorname{End}_{H}\left(X_{0}\right) \cong k$.

\section{REFERENCES}

[1] L. Angeleri Hügel And F. Coelho, Infinitely generated complements to partial tilting modules, Math. Proc. Camb. Phil. Soc. 132 (2002), 89-96. MR1866326 (2003i:16001)

[2] L. Angeleri Hügel, A. Tonolo and J. TrlifaJ, Tilting preenvelopes and cotilting precovers, Alg. Repr. Theory 4 (2001), 155-170. MR1834843 (2002e:16010)

[3] I.Assem, Torsion theories induced by tilting modules, Canad J. Math. 36 (1984), 899-913. MR762747 (86j:16022)

[4] I.Assem and O. Kerner, Constructing torsion pairs, J. Algebra 185 (1996), 19-41. MR1409972 (97f:16024)

[5] I. Assem, D. Simson and A. Skowroński, Elements of Representation Theory of Associative Algebras I: Techniques of Representation Theory, London Math. Soc. Studfent Texts 65, Cambridge Univ. Press, Cambridge 2006. MR2197389 (2006j:16020)

[6] M. Auslander, I. Reiten and S. Smalø, Representation Theory of Artin Algebras, Cambridge Stud. Adv. Math. 36, Cambridge Univ. Press, Cambridge 1994. MR1314422 (96c:16015)

[7] M. Auslander and S. Smalø, Almost split sequences in subcategories, J. Algebra 69 (1981), 426-454. MR617088 (82j:16048a)

[8] D. BAER, Wild hereditary artin algebras and linear methods, Manucripta Math. 55 (1986), 69-82. MR828411 (87i:16054)

[9] S.Bazzoni and D.Herbera, One dimensional tilting modules are of finite type.

[10] K. Bongartz, Tilted algebras, in Representations of Algebras, Lect. Notes in Math., Vol. 903, Springer, Berlin 1981, 17-32. MR654701 (83g:16053)

[11] S. Brenner And M.C.R. Butler, Generalizations of the Bernstein-Gelfand-Ponomarev reflection functors, in Representation Theory II, Lect. Notes in Math., Vol. 832, Springer, Berlin 1980, 103-169. MR607151 (83e:16031)

[12] R.Colpi And C.Menini, On the structure of *-modules, J.Algebra 158 (1993), 400-419. MR1226797 (94i:16003)

[13] R.Colpi And J.TrlifaJ, Tilting modules and tilting torsion theories, J.Algebra 178 (1995), 614-634. MR1359905 (97e:16003)

[14] W. Crawley-Boevey, Modules of finite length over their endomorphism rings, in Representation of Algebras and Related Topics, London Math. Soc. LNS 168, Cambridge 1992, 127-184. MR1211479 (94h:16018)

[15] W. Crawley-Boevey, Infinite dimensional modules in the representation theory of finite dimensional algebras, in Algebras and Modules I, Canad. Math. Soc. Conf. Proc., Vol. 23, AMS, Providence 1998, 29-54. MR1648602 (99m:16016)

[16] W. Geigle and H. Lenzing, Perpendicular categories with applications to representations and sheaves, J. Algebra 144 (1991), 273-343. MR1140607 (93b:16011)

[17] D. Happel and C.M. Ringel, Tilted algebras, Trans. Amer. Math. Soc. 274 (1982), 399443. MR675063 (84d:16027)

[18] D. Happel and L.Unger, Almost complete tilting modules, Proc. Amer. Math. Soc. 107 (1989), 603-610. MR984791 (90f:16026) 
[19] M. Hoshino, On splitting torsion theories induced by tilting modules, Com. Algebra 11 (1983), 427-439. MR689417 (85b:16028)

[20] O. Kerner, Tilting wild algebras, J. London Math. Soc. 39 (1989), 29-47. MR989917 (90d:16025)

[21] O. Kerner, Universal short exact sequences for torsion theories, in Topics in algebra, Banach Cent. Publ. Vol 26, PWN Polish-Scientific-Publishers, Warsow 1990, 317-326. MR1171241 (93h:16019)

[22] O. Kerner, Exceptional components of wild hereditary algebras, J. Algebra 152 (1992), 184-206. MR1190411 (94b:16018)

[23] O. Kerner, Representations of wild quivers, in Representation Theory of Algebras and Related Topics, Can. Math. Soc. Conf. Proc., Vol.19, AMS, Providence, 1996, 65-107. MR1388560 (97e:16028)

[24] O. Kerner and F. Lukas, Elementary modules, Math. Z. 223 (1996), 421-434. $\operatorname{MR} 1417853$ (98f:16011)

[25] O. Kerner and J. Trlifaj, Tilting classes over wild hereditary algebras, J. Algebra 290 (2005), 538-556. MR2153267 (2006d:16020)

[26] H. Krause, The spectrum of a module category, Memoirs Amer. Math. Soc. 707 (2001). MR1803703 (2001k:16010)

[27] H. Krause and M.SaOrin, On minimal approximations of modules, Contemp. Math. 229 (1998), 227-236. MR1676223 (99m:16002)

[28] F. LukAS, Infinite dimensional modules over hereditary algebras, J. London Math. Soc. 44 (1991), 401-419. MR1149004 (93b:16021)

[29] F. LukAs, Elementare Moduln über wilden erblichen Algebren, Ph.D. Thesis, Düsseldorf, 1992.

[30] C.M. RingeL, Finite dimensional hereditary algebras of wild representation type, Math.Z. 161 (1978), 235-255. MR501169 (80c:16017)

[31] C.M. Ringel, Infinite dimensional representations of finite dimensional hereditary algebras, Symposia Math. 23 (1979), Academic Press, 321-412. MR565613 (81i:16032)

[32] C.M. Ringel, Tame algebras and integral quadratic forms, Lecture Notes in Mathematics, Vol. 1099, Springer, Berlin, 1984. MR774589 (87f:16027)

[33] C.M. RingeL, The regular components of the Auslander-Reiten quiver of a tilted algebra, Chin. Ann. Math. 9B (1988), 1-18. MR943675 (89e:16036)

[34] H. Strauss, On the perpendicular category of a partial tilting module, J. Algebra 144 (1991), 43-66. MR1136894 (92m:16013)

[35] J. TrlifaJ, Cotorsion theories induced by tilting and cotilting modules, Contemp. Math. 273 (2001), 285-300. MR1817171 (2001m:16012)

Mathematisches Institut, Heinrich-Heine-Universität Düsseldorf, Universitätsstr.1, 40225 DÜSSELDORF, GERMANY

Faculty of Mathematics and Physics, Department of Algebra, Charles University, Sokolovská 83, 18675 Prague 8, Czech Republic 